\title{
Proximal Femoral Nail in Intertrochanteric Femoral Fractures
}

\author{
Mehboob I' \\ 'Department of Orthopaedic Surgery, SIMS / Services Hospital Lahore, Pakistan.
}

\section{ABSTRACT}

Introduction: Proximal femoral nails (PFN) have been introduced recently but have begun to compete the traditional dynamic hip screw. The mechanical strength of the nail and less invasive procedure has made the procedure preferable. This is a short retrospective review of 26 cases operated in the last two years.

Methods: A retrospective review was conducted of 26 cases operated during two years of study which had completed at least a year of follow up. All had been treated using a proximal femoral nail for unstable intertrochanteric fractures of femur. A radiological assessment was made with serial X-rays.

Results: The operating time was found to be short, less blood loss was seen during surgery and few early complications were noted. All cases were relatively free from long term complications.

Conclusions: PFN is a suitable implant for unstable intertrochanteric femoral fractures needing open reduction internal fixation. It has low per operative and post operative morbidity.

Key Words: femur, fracture, intertrochanteric, nail, proximal

\section{INTRODUCTION}

Fracture fixation with dynamic hip screw (DHS) has been the gold standard, but new implant like intramedullary nails have recently challenged DHS as the best method of treatment for intertrochanteric fractures. ${ }^{1}$ Plate constructs that provide biaxial dynamic compression, lag screws with improved purchase, devices with multiple lag screws, and those designed for percutaneous insertion are among the many recent innovations to compression hip screw design. The proximal femoral nail (PFN) is a recently introduced device. ${ }^{2}$ Lesser exposure along with smaller operating time and less blood loss are other advantages that a PFN implant has over the conventional DHS. ${ }^{2-5}$ Patients treated with an intramedullary nail have been found to have shorter operative times, fewer blood transfusions, and shorter hospital stays compared with those treated with a $95^{\circ}$ screw-plate.
Correspondence:

Dr. Irfan mehboob

29 XX, DHA Lahore, Pakistan.

Email: awanortho@yahoo.com

Phone: 92-333-430-0901 


\section{METHODS}

This is a retrospective, multicentered study done at two institutions, Postgraduate Medical Institute, Lahore and Sindh Government Lyari General Hospital, Karachi from February, 2005 - February, 2007 for unstable fractures of proximal femur using the PFN. Hospital record files were used to chose the patients who were called back for review if needed. Ethical approval was taken from the ethical committee of the hospital and data was analyzed using the SPSS version 14 . Serial X-rays if available were used to assess progress of union postoperatively. Only cases, where X-rays, at least 1 year after surgery were available for review or the patient who could come back for review were included (Figure $1)$.

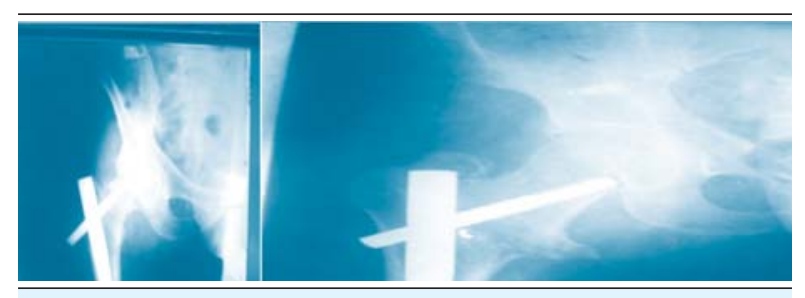

Figure 1. Radiographs of two cases taken six weeks after surgery.

\section{Operative technique}

All were operated under C-arm. The patients were put in supine position with the affected side up. A small incision over the greater trochanter area was made and the tip of greater trochanter was exposed and entry point made by using the femoral awl. Using the guide wire and the cannulated reamers the proximal femur was reamed. Having seated the nail in the proximal femur the compression screw was introduced. The distal interlocking screws were placed through the jig provided with the instrumentation. The final position was checked under the image intensifier. The patients were given intra venous antibiotics as prophylaxis. All were encouraged to start non weight bearing crutch mobilization after 24 hours of surgery. Low molecular weight heparin was used to prevent deep vein thrombosis. All were discharged on second post operative day except when needed for other compelling reasons.

In out patient serial X-rays were taken at monthly intervals and gradual weight bearing was started around 6 weeks after surgery.

\section{RESULTS}

In all 32 patients, record was available for review but only 26 were on follow up. All patients who had been operated had returned to their work or pre-injury status.
There was radiological union accompanied by appropriate clinical findings. The average age of the study population was 57.24 years (range: $35-70$ years). Total $10 / 26$ were injured in a road traffic accident and rest had domestic fall. One patient developed superficial wound infection which settled with intra venous antibiotics selected as per culture studies. Three patients had displacement of antirotation screws during follow up at some stage $(11.53 \%)$. One patient complained of pain over the trochanter area during follow up. There was one case of deformation of trochanteric screw after weight bearing. All were ambulant at the time of final compilation of results (Table 1 ).

\section{DISCUSSION}

The findings of Miedel indicate that the Gamma Nail showed good results in both trochanteric and subtrochanteric fractures. ${ }^{6}$ Incidence of technical failure in patients with unstable trochanteric fracture was $6.5 \%$ (6/93) in the Standard Gamma Nail group and $5.2 \%$ $(5 / 96)$ in the Medoff Sliding Plate group. ${ }^{6}$ Patients treated with an intramedullary nail have had shorter operative time, fewer blood transfusions, and shorter hospital stay compared with those treated with a $95^{\circ}$ DHS. ${ }^{1}$ Our operation time is far less than quoted by other studies. In the study by Menezes, out of 129 patients available for follow-up failure of fixation occurred in three patients $(2 \%)$, and a femoral shaft fracture occurred in one patient $(0.7 \%){ }^{4}$ No such event has been noted by us as yet but our follow up is very short, as time passes by we may note other problems. Fixation failures included one cutout, one delayed fracture healing, and one lateral displacement of the antirotation screw. In other studies total re-operation rate was high (12\%) mainly because of hardware removals, which occurred in 13 patients $(8.6 \%)$. Stratification of results showed that hematomas and iliotibial tract irritation occurred more commonly with lesser surgical experience. ${ }^{4}$ We have a very small group of patients hence cannot comment on problems related to learning curve of the procedure but we have noted our operating time decreased with increase in experience. Generally, the results of treatment of unstable trochanteric fractures were comparable for the PFN and Gamma Nail. The pitfalls and complications were similar, and mainly surgeon or fracture-related, rather than implant-related. ${ }^{2}$

Our operating time was short and we did not have to transfuse blood post operatively. In other studies the intra-operative blood loss was lower with the PFN (220 $\mathrm{ml} \vee 287 \mathrm{ml}, \mathrm{p}=0.001){ }^{2}$ We did not make any direct measurements of blood lost during surgery and accept this as a limitation of our findings. Post-operatively, more lateral protrusion of the hip screws of the PFN $(7.6 \%)$ was documented, compared with the gamma nail $(1.6 \%, p=0.02)$ in the series published by Schipper. ${ }^{2}$ 
Table 1. Results compiled 18 months after surgery

\begin{tabular}{ccr}
\hline Time of Operation & \multicolumn{1}{c}{$40-90 \mathrm{~min}$} & $61.64 \mathrm{~min}$ \\
\hline Blood Transfusion Pre-OP & 3 & $11.53 \%$ \\
Post-OP & 1 & $3.84 \%$ \\
Period of healing of fracture & $12-20$ weeks & 15.4 weeks \\
Complications Infection & 1 & $3.84 \%$ \\
Anti-rotation screw & 3 & $11.53 \%$ \\
Trochanteric Bursitis 1 & $3.84 \%$ \\
Deformation & 1 & $3.84 \%$ \\
Shortening $>2 \mathrm{~cm}$ & 2 & $7.68 \%$ \\
\hline
\end{tabular}

Most local complications were related to suboptimal reduction of the fracture and/or positioning of the implant. ${ }^{2}$ Functional outcome and consolidation were equal for both implants. Results of treatment of unstable trochanteric fractures were comparable for the PFN and Gamma Nail. ${ }^{2}$ One case of trochanteric bursitis was noted but we failed to find any gross lateral displacement of femoral screw in the later X-rays of this case.

\section{CONCLUSIONS}

Though small scale and of rather short duration of follow up, our findings show that PFN has advantages over the conventional Dynamic Hip Screw. A shorter operating time, less blood loss, easy operative technique and lesser post operative problems point to the promise the implant shows. There is need for more randomised controlled trials.

\section{ACKNOWLEDGEMENTS}

We are thankful to those who contributed in designing, Dr. Irfan Mehboob and implant manufacturing, Osteomet \& Co, Dr Faisal Nazeer for data research, Dr. Parvaiz Anjum for statistical analysis and those who were involved in data collection and literature search.

\section{REFERENCES}

1. Sadowski C, Lübbeke A, Saudan M, Riand N, Stern R, Hoffmeyer P. Treatment of reverse oblique and transverse intertrochanteric fractures with use of an intramedullary nail or a 95 degrees screw-plate: a prospective, randomized study. J Bone Joint Surg Am. 2002 Mar;84-A(3):372-81.

2. Schipper IB, Steyerberg EW, Castelein RM, van der Heijden FH, den Hoed PT, Kerver AJ et al. Treatment of unstable trochanteric fractures. Randomised comparison of the gamma nail and the proximal femoral nail. J Bone Joint Surg Br. 2004 Jan;86(1):86-94.

3. Pajarinen J, Lindahl J, Michelsson O, Savolainen V, Hirvensalo E. Pertrochanteric femoral fractures treated with a dynamic hip screw or a proximal femoral nail. A randomised study comparing post-operative rehabilitation. J Bone Joint Surg Br. 2005 Jan;87(1):76-81.
4. Menezes DF, Gamulin A, Noesberger B. Is the proximal femoral nail a suitable implant for treatment of all rochanteric fractures? Clin Orthop Relat Res. 2005 Oct;439:221-7.

5. Mattsson P, Alberts A, Dahlberg G, Sohlman M, Hyldahl HC, Larsson S. Resorbable cement for the augmentation of internallyfixed unstable trochanteric fractures. A prospective, randomised multicentre study. J Bone Joint Surg Br. 2005 Sep;87(9):1203-9.

6. Miedel R, Ponzer S, Törnkvist H, Söderqvist A, Tidermark J. The standard Gamma nail or the Medoff sliding plate for unstable trochanteric and subtrochanteric fractures: A randomised, controlled trial. J Bone Joint Surg Br. 2005 Jan;87(1):68-75. 